\title{
Restricción de Crecimiento Intrauterino. Causas, Características Clínicas, y Evaluación de Factores Asociados a Policitemia Sintomática
}

\author{
GLADYS M. GODOY TORALES ${ }^{1}$, MABEL ZACUR DE JIMÉNEZ ${ }^{2}$ \\ 1. Médico Pediatra. Jefe de Sala. Servicio de Neonatología. Hospital Nacional de Itauguá. \\ 2. Gastroenteróloga Pediatra. Docente de la Cátedra de Pediatría. Facultad de Ciencias Médicas. Jefe de Sala Hospital.
}

\begin{abstract}
Intrauterine Growth Restriction: Causes, Clinical Characteristics, and Evaluation of Factors Associated with Symptomatic Polycythemia
\end{abstract}

The incidence of newborns (NB) with intrauterine growth restriction (IUGR) in Latin America and the Caribbean is $10 \%$. Objectives: To determine the causes and characteristics of IUGR in children born at the Hospital Nacional in Paraguay from January 1999 to December 2001 and establish which factors are associated with symptomatic polycythemia. Materials and Methods: An observational and descriptive retrospective cohort study with nested case-controls including 259 NB with birth-weight below the 3d percentile. Results: 165 $(64 \%)$ of NBs were premature, with a mean age of $33.1 \pm 3.1$ weeks. $220 \mathrm{NB}(84.9 \%)$ showed symmetrical IUGR and 37 (14.2\%) perinatal asphyxia. The most frequent causes of IUGR were maternal hypertension 87 $(33.5 \%)$, adolescent mother $67(25.9 \%)$, older mothers $44(17 \%)$, twin pregnancy $34(13 \%)$, and STORCH group infections 17 (6.5\%). The dominant pathologies in NBs were hyperbilirubinemia $119(47 \%)$, transitory tachypnea $76(30 \%)$, hyaline membrane disease $49(19 \%)$, nosocomial sepsis $43(17 \%)$, and hypoglycaemia $14(36 \%)$. Of the $195 \mathrm{NB}$ with hematocrit (HCT), 53 (27\%) developed polycythemia, and of those, $14(26 \%)$ were symptomatic. Symptoms included hypoglycemia $6(43 \%)$, weak suck $6(43 \%)$, apneas $4(18 \%)$, respiratory distress and hypotonia $3(21 \%)$. The NB with symptomatic polycythemia had mostly suffered asphyxia $(\mathrm{OR}=2.92)$, had asymmetric IUGR $(\mathrm{OR}=2.39)$, or been children of preeclamptic mothers $(\mathrm{OR}=1.73)$. NB with thrombocytopenia were least frequently symptomatic. No significant difference was shown in mean HCT between symptomatic and asymptomatic NB. Conclusions: The most common cause of IUGR was maternal arterial hypertension (AHT). More than half of NB with IUGR were premature, with the symmetrical form being more common. Factors associated with evolution of polycythemia were NB who were asphyxiated or with preeclamptic mothers.

(Key words: Fetal growth retardation, etiology, risk factors, symptomatic polycythemia).

Pediatr. (Asunción), 2008; 35(2): 77-87 


\section{RESUMEN}

La incidencia de recién nacidos (RN) con restricción de crecimiento intrauterino (RCIU) en países de América Latina y el Caribe es del $10 \%$. Objetivos: Determinar las causas y características de RCIU, en los niños nacidos en el Hospital Nacional, Paraguay, desde enero de 1999 a diciembre del 2001. Establecer los factores asociados al desarrollo de policitemia sintomática. Material y Método: Estudio observacional, descriptivo, de cohorte retrospectivo; y caso-control anidado. Se incluyeron $259 \mathrm{RN}$ con peso de nacimiento < percentil 3 . Resultados: 165 (64\%) de los RN fueron prematuros, con una media de edad de 33,1 $\pm 3,1$ semanas. $220 \mathrm{RN}$ (84,9\%) tuvieron RCIU simétrico, y $37(14,2 \%)$ asfixia perinatal. Las causas más frecuentes de RCIU fueron la hipertensión materna $87(33,5 \%)$, madre adolescente $67(25,9 \%)$, madre añosa $44(17 \%)$, embarazo gemelar $34(13 \%)$, e infecciones del grupo STORCH en $17(6,5 \%)$. Las patologías preponderantes en los RN han sido hiperbilirrubinemia $119(47 \%)$, taquipnea transitoria $76(30 \%)$, enfermedad de membrana hialina $49(19 \%)$, sepsis intrahospitalaria 43 (17\%), e hipoglucemia en 14 (36\%). De los $195 \mathrm{RN}$ que contaban con hematocrito (Hto), 53 (27\%) desarrollaron policitemia; y de ellos $14(26 \%)$ fueron sintomáticos. Los síntomas fueron: hipoglucemia $6(43 \%)$, succión débil $6(43 \%)$, apneas $4(18 \%)$, distress respiratorio e hipotonía en $3(21 \%)$. Los RN con policitemia sintomática fueron en su mayor proporción asfixiados $(\mathrm{OR}=2,92)$, con RCIU asimétrico $(\mathrm{OR}=2,39)$, e hijos de madres pre-eclámpticas $(\mathrm{OR}=1,73)$. Los $\mathrm{RN}$ con trombocitopenia menos frecuentemente fueron sintomáticos. No existió diferencia significativa en la media de Hto de los RN sintomáticos y asintomáticos. Conclusiones: La causa más frecuente de RCIU fue la HTA materna, más de la mitad de los RN con RCIU fueron prematuros, la forma simétrica fue la más frecuente. Los factores asociados al desarrollo de policitemia fueron: $\mathrm{RN}$ asfixiados e hijos de madres pre-eclampticas.

(Palabras clave: Retardo del crecimiento fetal, etiología, factores de riesgo, policitemia sintomática). Pediatr. (Asunción), 2008; 35(2): 77-87

ESTE TRABAJO LO PUEDE ENCONTRAR EN EXTENSO EN WWW.SciELO.ORG 